dei Tartari fiori il commercio che era soprattutto nelle mani degli italiani, dei greci e degli armeni. Accanto a Sarai, fungevano da centri commerciali i porti della Crimea e delle foci del Dnjestr e del Don. La popolazione dell'Orda d'oro vien valutata a 300000 ed etnograficamente doveva possedere una struttura molto complessa. Ancor oggi si riscontrano gli influssi esercitati dal regno dei Tartari: l'orientamento dell'Oriente su Bisanzio creò le premesse per il contrasto culturale tra l'Europa Orientale e Occidentale.

\title{
KARTOGRAPHIE IN AMERIKA
}

\section{Berthold Carlberg}

Das Department of Social Affairs der United Nations hat erstmalig 1951 einen nunmehr für jedes Jahr vorgesehenen Bericht veröffentlicht über den Stand und die Fortschritte der Kartographie in der Welt 1 . Der nicht amerikanischen Fachwelt wird damit ein willkommener Überblick vermittelt über die zum wesentlichen Teil durch die Forderungen des Krieges veranlaßte außerordentliche Entwicklung der kartographischen Praxis und Technik, zu deren weiterer Förderung nun nach dem Kriege noch ein Anstoß gekommen war durch die Nutzbarmachung der in nicht minder angestrengten Bemühungen auf der «Gegenseite 》 gemachten Fortschritte. Es dürfte deshalb lohnen, nicht nur für den Kartographen, sondern auch für den Geographen und verwandte Disziplinen, sich an Hand dieser Schrift einführen zu lassen in das Gesamtgebiet der Kartographie, die hier nach der von dem Komitee der Experten für Kartographie vom April $1949^{2}$ gegebenen Definition als jene Wissenschaft verstanden wird, "die alle Arten von Land- und Seekarten bearbeitet. Sie schließt jede Tätigkeit ein von der Vermessung bis zum Druck der Karte ».

Der Titel der Veröffentlichungen mu $\beta$ allerdings insofern als ein zunächst lediglich programmatischer genommen werden, als sich die Berichterstattung dieses ersten Bandes ausschließlich auf amerikanische, insbesondere auf vereinsstaatliche Verhältnisse beschränkt. Sie bildet damit in gewisser Weise eine Ergänzung zu des Referenten Aufsatz über «Kartographie in den USA » ${ }^{3}$, der jedoch mehr auf wissenschaftlich-kartographische und insbesondere auf privat-kartographische Fragen eingegangen war, während die vorliegende Schrift ausschließlich die 'Tätigkeit der amtlichen Kartographie berücksichtigt, diese allerdings in ihrem weitesten, oben abgesteckten Umfange.

Der die «Tätigkeitsberichte» einleitende Aufsatz über «Kartographie in den Amerikas 》 gibt eine entwicklunsgeschichtliche Darstellung vom Zustandekommen der "Commission on Cartography 》 im Rahmen des Panamerican Institute of Geography and History, dem die Initiative zu der vorliegenden Veröffentlichung zu verdanken ist. Nach einem schon 1903 von Seiten verschiedener südamerikanischer Staaten gemachten Vorschlag ist sie 1941 gegründet worden mit dem $Z$ wecke der Förderung und Koordinierung ihr verwandter Studien. Hierzu bilden eine alle 4 Jahre stattfindende Generalversammlung (1943 in Washington, 1944 in Rio, 1946 in Caracas, 1948 in Buenos Aires und 1949 in Santiago), ein Verwaltungsausschu $\beta$ mit Sitz in Mexico City und 3 Kommissionen, von diesen eine die für Kartographie, den Rahmen. Das Aufgabengebiet dieser "Commission on Cartography 》 ist schon durch die Unterabteilungen umrissen: der der Geodäsie, die ihrerseits Schweremessung, Erdmagnetismus und Erdbebenforschung einbegreift, und der der Topographischen Karten und der Photogrammetrie, denen noch Fliegerkarte, Seekarte, Gezeiten, Spezialkarten und Stadtpläne zugewiesen sind.

1 World Cartography, Volume I, 1951. United Nations, Department of Social Affairs, New York.

2 Vgl. Modern Cartograply, Base Maps for World Needs. U. N. Publication sales Nr. 1949.

I. 19. S. 7, vgl. den Artikel hierüber von F. FluRy, Geographica Helvetica VI, 1951, p. 276-278.

${ }^{8}$ Petermanns Mitteilungen 1950, II, S. 113-118. 
Welche Bedeutung Vermessung und Kartierung, auf amerikanische Verhältrisse gesehen, auch heute noch zukommen, erhellen folgende, der vorliegenden Schrift entnommene Tatsachen. Erfährt man doch da u. a. von einem auf $200 \mathrm{~km}$ sich erstreckenden, an die $1600 \mathrm{~m}$ hohen Gebirge, keine $100 \mathrm{~km}$ vom Panamakanal entfernt, das noch auf keiner Karte verzeichnet ist. Erfährt man weiter, daß Gipfelhöhen in den Karten bis zu $50 \%$ falsche Werte haben, daß, wie neuerdings festgestellt wurde, die Isle of Pines, ein Hauptansteuerungspunkt für den Flughafen von Habana, wahrscheinlich um $20-30 \mathrm{~km}$ von ihrer wirklichen Position entfernt eingetragen ist.

Neben ihren Hauptaufgaben, der Aufstellung von Standardrichtlinien für die verschiedenen Kartenreihen der amerikanischen Länder, dem Berichtaustausch auch über technische Neuerungen, Schulung des aufnehmenden und zeichnenden Personals, hat sich die Commission on Cartography mit der Herausgabe einiger instruktiver Filme über Vermessung und Kartierung befaßt, hat das jüngst erschienene «Glossary of Cartographic Terms 》 (Februar 1948) herausgebracht und unterstützt nicht zuletzt auch die Fortführung der Arbeiten an der Map of Hispanic America 1:1 Mill. der Am. Geogr. Society.

«Tätigkeitsberichte » liegen vor von Brasilien, Canada und den USA. Davon befaßt sich der von Brasilien nach einer historischen Einleitung, aus der hervorgeht, daß die beiden Hauptinstitute, der Conseilho Nacional de Geografía und der Serviço Geografico del Exèrcito mit Gesetz vom April 1946 nach gemeinsamem Plan zusammenarbeiten, mit der Aufzählung dieser Aufgaben: neben den Triangulierungen I. Ordnung die Fortführung der Topographischen Karten 1:50 000 neben $1: 25000,1: 100000$ und $1: 250000$.

Canada hat sich in der Kartierung seiner weiten Gebiete ganz auf Flugbildaufnahmen eingestellt, für die Tiefenlotung auf das Echolot. Geodätische Messungen werden seit einigen Jahren mit Radar (Shoran) durchgeführt, und der Einsatz des Helicopters ermöglicht es, in schwierigstem Terrain zu arbeiten. Hauptträger dieser Aufgaben ist der Geodetic Survey of Canada, der 1905 eingerichtet worden ist. Von Interesse ist das Ergebnis eines Höhennivellements vom Pazifik zum Atlantik hinüber, das eine Niveaudifferenz von nur $1 \mathrm{Fu} \beta(0,3 \mathrm{~m})$ ergab.

Die Vermessungs- und Kartierungstätigkeit $\operatorname{der} U S A$ steht unter dem Zeichen einer gewaltigen Erweiterung ihrer Aufgaben. Ihr Umfang ist seit 10 Jahren auf. das 7 fache gestiegen. Hinzu kommt eine durchgreifende Umstellung in der Technik der Verfahren, indem auch hier der Meßtisch vom Luftbild abgelöst wurde. Der Coast and Geodetic Survey überwacht die Dreiecksnetze höherer Ordnung, der Geological Survey erstellt die Topographischen Karten und schafft zudem auch $80 \%$ der Topographischen Aufnahmen. Daneben befaßt sich der Army Map Service des Corps of Engineers hauptsächlich mit der Kartierung auswärtiger Gebiete. Schließlich haben auch Flotte und Luftwaffe noch ihre eigenen Institute, das Hydrographic Office und den Aeronautical Chart Service. Nach Einführung der Luftbildvermessung im Jahre 1920 sind heute $50 \%$ der USA photogrammetrisch aufgenommen. Im Maßstab 1:62 500 sind heute vom Geol. Surv., der seit 1879 arbeitet, etwa $10 \%$ der Staaten kartiert.

Der Umfang der Aufgaben mag an einer Aufzählung der wichtigsten von diesem Institut betreuten Kartenwerke deutlich werden: neben den Maßstäben 1:24000, 1:31680, 1:63500 und 1:250 000 für den privaten Bedarf, die militärischen Zwecken dienenden in 1:25000, 1:50000, 1:100 000. Dazu Bodenklassierungskarten in 1:125000 und 1:250000 mit Detailaufnahmen in $1: 31800$ und $1: 15840$.

Der Hydrographic Survey, 1835 gegründet, bringt Segelkarten (1:600 000 und kleiner), Generalkarten $(1: 100000$ bis $1: 60000)$. Küstenkarten (1:50000 bis 1:100000) und Hafenpläne (größer als 1:50 000) heraus, dazu die Intercostal Waterway Charts in 1:40000.

Fliegerkarten erscheinen als Sectional Charts (1:500 000), World Aeronautical Charts (1:1 Mill.), als sog. Strip Charts, die sich auf 100 Meilen zuseiten einer bestimmten Flugstrecke ausdehnen, Local Charts 1:250 000, dazu Einflug- bzw. Landekarten in größeren Maßstäben. 
Was die vorliegende Veröffentlichung besonders wertvoll macht, sind neben diesen Berichten die «Technischen Notizen und Studien». Ihnen geht voraus ein allgemeiner Aufsatz über «Die Kartographie in ihrer Bedeutung für die ErschlieBung von Rohstoffquellen » von R. H. RANDALL und A. J. SwEET, in dem ausführlich ihre Aufgaben für die Wasserversorgung, Hochwasserkontrolle, Bewässerung, für die Förderung der landwirtschaftlichen Erzeugung, Drainage, Kraftwerke, Kanäle für Bergbau bis hin zu Fischereischutz und Wildhege entwickelt werden. Es folgt dann noch eine vermessungstechnische Studie von W. SchermerHORN, Leiter des Internation. Schulungsamts für Luftaufnahmen, über «Bodenkontrolle als Engpaß bei der Luftaufnahme» Verf. ist der Auffassung, entgegen anderer Meinung von Fachgenossen, welche Lufttriangulation nur für gerechtfertigt erachten bei ungewöhnlichen, etwa militärischen Aufgaben von entsprechend geringeren Genauigkeitsanforderungen, daß die Aerotriangulation zur allgemeinen Einführung kommen wird, wenn sich die Photogrammeter erst mit ihr vertraut gemacht haben. Zudem stehen über die Verwendungsmöglichkeiten der Radarverfahren (Shoran und Decca) Erfahrungen noch aus, die weitere Kontrollmöglichkeiten schaffen werden.

Es ist sehr dankenswert, daß dann Cl. A. Burmister ( mittel für Vermessung und Kartierung ») einen Überblick gibt über die verschiedenen Systeme der elektronischen, bezw., da den verwendeten Methoden allen das Radar- (Radio Direction and Range) Prinzip zugrunde liegt, der Radar-Navigation.

Von ihnen verwenden Shoran (short range navigation), Loran (long range navigation) und der EPI (Electronic position indicator) elektromagnetische Impulse, sehr kurze und kräftige Stromstöße, während die (britischen) Systeme der Decca, Raydist, Lorac u. a. mit kontinuierlichen Wellen arbeiten. Bei Loran steht das Flugzeug bzw. das Schiff mit 2 Paaren von Bodenstationen in Verbindung, d. h., da eine Station beiden gemeinsam sein kann, mit 3 Stationen. Diese sind untereinander synchronisiert, senden jedoch ihre Impulse zeitlich um ein geringes gegeneinander verschoben. Der Empfänger stellt die zeitliche Differenz in der Ankunft der von den verschiedenen Stationen gegebenen Signale fest und kann daraus die Entfernung zu den Stationen ermitteln. Die Sonderkarten, die neuerdings zum $Z$ wecke der Loran-Navigation hergestellt werden, sind dazu mit solchen Linien gleicher Differenzen ïberzogen, bei Loran Hyperbelscharen mit den Brennpunkten in den jeweiligen Bodenstationen. Die Schnitte der ermittelten Differenzlinien geben den Standort des Schiffes (Flugzeuges). Das Shoransystem arbeitet mit 2 Stationen, die Positionslinien sind hier um die Stationen gezogene konzentrische Kreise.

$\mathrm{Da}$ die elektromagnetischen Wellen eine Geschwindigkeit von $30 \mathrm{~cm}$ pro Mikrosekunde $(300$ $\mathrm{km}$ pro Sekunde) haben, sind feinste Zeitmessungen möglich, die beim Shorangerät bis zu 0,01 Mikrosekunden gehen bei einer Ablesegenauigkeit von $0,1 \mathrm{~m}$. Man rechnet bei Shoran mit Strekkenfehlern von maximal $8 \mathrm{~m}$, bei längeren Distanzen von $16-25 \mathrm{~m}$. Während Shoran Strecken bis zu $800 \mathrm{~km}$ zwischen 2 Bodenstationen messen kann, geht die Reichweite von Loran - bei entsprechend verringerter Genauigkeit - bis zu $1120 \mathrm{~km}$, bei Nacht bis zu $2400 \mathrm{~km}$.

Loran und Decca dienen mit ihren längeren Reichweiten vorwiegend der Navigation, Shoran und EPI als die genaueren (aber auch mit geringerer Reichweite) sind mit Erfolg für geodätische Vermessungen eingesetzt worden. Werden diese erst seit einigen Jahren entwickelten Methoden schon von revolutionierender Wirkung auf das Vermcssungswesen sein, so ist zu bedenken, daß auch die Einführung der Aerophotogrammetrie jüngeren Datums ist, in den Staaten damit erst 1920 begonnen wurde. Ein Menschenalter also, da der Topograph noch mit dem Meßtisch auf dem Rücken bezw. auf seinem Packtier auf die $W$ anderschaft ging, wo ihm heute die Bildkamera, Radio und Radar, Helicopter und Amphibienfahrzeuge die Arbeit erleichtern, dabei schnellere und genauere Resultate erzielen.

Der Aufsatz von G. D. Whitmore und M. M. 'Thompson über « einige neue Fortschritte in der Kartographie » und ein folgender Auszug aus «Progress Report on the Cartographic Activities of the USA for the Period from 1st July 1948 to June 50» mit dem Titel «Notizen über neue Fortschritte in der kartographischen Ausrüstung und Technik in den USA » führen die wesentlichsten Neuerungen auf den genannten Gebieten auf. 
Schon ist die Höhenmessung mit Radar beispielsweise soweit entwickelt, daß man nach dem Echolotprinzip ein durchlaufendes Profil längs der Fluglinie herstellen kann. Und möge auch die Genauigkeit noch durch die Höhenschwankungen des Flugzeugs beeinträchtigt sein, so hat man schon geeignete Kontrollapparate an der Hand, um diese Fehler auszugleichen. Ein Höhenmesser ganz anderer Art wird dann erwähnt, der mit einem auf einem Dreiradfahrzeug montiertem sehr empfindlichen Pendel arbeitet. Dieses löst elektrische Signale aus, deren Stärke sich mit dem sin des Hangwinkels verändert. Gekoppelt mit einem Kilometerzähler, vermag das Gerät Höhenprofile herzustellen, deren Fehler $2 / 3 \mathrm{~m}$ nicht überschreiten. Oder man erfährt von einem einfachen Gerät, wie es dem Höhlenforscher an die Hand gegeben ist, um damit die Deckenhöhen von Höhlen zu messen, einem mit Wasserstoffgas gefüllten Ballon, der eine Meßmarke mit hoch nimmt.

Ein Problem war bisher noch die Vermessung flacher und unzugänglicher Küstenstrecken. Heute werden neben Helicoptern Amphibienfahrzeuge (DUKW) eingesetzt, um kleinere Arbeitsgruppen mit Gerät ohne Umsteigen vom Schiff aufs Land zu schaffen. Flachwasserboote von nur $30-35 \mathrm{~cm}$ Tiefgang bei voller Ladung von Gerät und 6 Mann Besatzung lassen Arbeiten in seichten Gewässern zu. Für Triangulierungen III. Ordnung hat man an unzugänglichen Küsten den landing ship tank (LST) eingesetzt, dessen Besatzung mit mehreren Arbeitsgruppen am Lande zusammen arbeitet und dabei durch Radio mit ihnen in Verbindung steht. Mit Hilfe von Shoran ist weiterhin heute die Möglichkeit gegeben, Tiefenlotungen bis zu 50 und 75 Seemeilen Entfernung von der Küste ihrer Position nach festzulegen. So stehen für die Küstenvermessung neue und schnellere Methoden bereit, um damit dem Dilemna abzuhelfen, daß noch weite Küstenstrecken bis heute kompiliert wurden aus über 100 Jahre alten Grundlagen britischer und spanischer Seeleute.

In der Luftphotogrammetrie spielt heute neben andern Weitwinkelkameras die Trimetrogonkamera die Hauptrolle, vor allem für Übersichtskarten 1:250000. Geräte sind hier in Erprobung, um Kontrollpunkte II. Ordnung aus Trimetrogonphotos festzulegen. Für den genannten Maßstab werden die (Weitwinkel-) Aufnahmen in $10000 \mathrm{~m}$ Höhe durchgeführt, ihre Auswertung im Weitwinkel-Multiplex gemacht. Für derartige Höhen steht noch eine Sonder-Kamera zur Verfügung von $6,1 \mathrm{~m}$ Brennweite. Sie ist in einem Spezialflugzeug untergebracht. Die Brennweite ist durch Prismen in drei Segmente zerlegt worden. Wieder eine andere Kamera, die «T 9 », ist für Aufnahmen aus schnell fliegenden Flugzeugen (bis zu 1600 $\mathrm{km} / \mathrm{std}$.) bestimmt. Mit ihr kann, bei elektrisch vollautomatischer Betätigung ein fortlaufender Bildstreifen hergestellt werden.

Neben dem Gerät des Photogrammeters ist auch das des Topographen verbessert worden. Hier ist es vor allem die Ausstattung der Theodoliten mit photographischer Ablesung, die über lange Distanzen noch gute Resultate sichert. Die Vorzüge des Leichtmetalls in bezug auf Transport und Handhabung haben zur Fertigung der verschiedensten Instrumente aus diesem Metall geführt, von Vermessungsniarken angefangen über Signallampen und Chronographen bis zu den trigonometrischen Turmbauten, die heute in zwei Größen zu 7,62 m und 33,53 m, letztere bei einem Gewicht von $3002 \mathrm{~kg}$ zur Verfügung stehen. Sie sind für Packtiertransport wie für Flugzeugabwurf zerlegbar konstruiert.

Auch das Gerät für die Bildauswertung hat mancherlei Vervollkommnung erfahren. Der normale Zeiß-Aeromultiplex ist jetzt auch für Schrägaufnahmen verwendbar gemacht worden, wobei Trimetrogonbilder mit einer Genauigkeit von $1 / 400$ der Flughöhe ausgewertet werden. Dem Multiplex ähnlich ist der KelshAuswerter entwickelt worden, der gegenüber der 2,4-fachen beim Multiplex eine 5 - fache Vergrößerung zuläßt. Speziell für Schrägaufnahmenauswertung ist der «Twinplex», bestehend aus 2 Weitwinkel-Multiplex-Projektoren, entstanden. Vom Zeiß-Stereoplanigraphen, der normalerweise für verschiedene Bildformate verwendbar, zur besseren Ausnützung ausschließlich auf die Standardmasse des Army Map Service $(22,86 \times 22,86 \mathrm{~cm}$ bei $15,24 \mathrm{~cm}$ Brennweite) umgestellt wurde, 
ist ein kleineres Modell gebaut worden für den Gebrauch bei kleineren Vermessungstrupps, auf Hochschulen usw. Auch das Prinzip des «Luz» (Luftbildumzeichner) findet sich in mehreren Modellen wieder, so im «Multiscop 》 und im « Fairchild Rectoplanigraph » zur Übertragung von Luftbildern in das Kartenmanuskript.

Die bisher gemachten Erfahrungen haben übrigens dazu geführt, bei Neuaufnahmen zwei in verschiedenen Höhen geflogene Bildreihen des gleichen Gebietes aufzunehmen, deren eine, aus größeren Höhen, für die horizontale Bodenkontrolle, die andere für Höhenlinien und topographisches Detail ausgewertet wird.

Eine eigene Wissenschaft, die Photogeologie, befaßt sich mit der Luftbildinterpretation für geologische Studien. Hier ist man dabei, Versuche mit Farbphotos zu machen. Daß auch der Infrarot-Film neue Möglichkeiten bietet, haben Versuche der Forstverwaltung gezeigt, die sowohl Infrarotfilm (mit Minus-Blau-Filter, Kodakgelb Nr. 12) verwendet wie panchromatischen Film mit dem gleichen Filter: wobei letztere sich vorwiegend für Nadelwald, der andere für Gemischtwald geeignet zeigte.

Zeichnung und Kartendruck sind durch die Einführung der durchscheinenden, maßhaltigen Plastikfolien (Vinylit u. a.) grundlegend in neue Bahnen gewiesen worden. Man ist jetzt dabei, auch Photofilm auf Vinylbasis herzustellen, der, stärker als der normale Photofilm $(0,18-0,20 \mathrm{~mm})$ diesem gegenüber eine kaum merkbare Schrumpfung aufweist, bei $122 \times 122 \mathrm{~cm}$-Folien nur $0,2 \mathrm{~mm}$ und dieses gleichmäßig über die ganze Fläche hin. Auf der anderen Seite verwendet man dehnbaren Dünnfilm, um ohne Zeichenarbeit Kartenteile aus einer Projektion in eine andere zu übertragen. Die Karten werden auf Dünnfilm photographiert, auf der Rückseite mit einem Haftmittel versehen und dann stückweise in das neue Netz eingepaßt. Da das bisher gebrauchte Material (Kodapack und Zellophan) zum Einkleben von Schrift und Signaturen den Nachteil hatte, Zeichentusche schlecht anzunehmen, sie z. T. auch keine genügende Schwärze für die Kopie aufwiesen, ist ein neuer Film entwickelt worden, matte-surface celanese, der frei von diesen Mängeln ist." Man kann auf ihm daher auch nach dem Einkleben der Namen usw. die Zeichnung vervollständigen.

Schon während des Krieges ergab sich die Notwendigkeit, zur besseren Veran. schaulichung der Bodenformen die Karten mit einem Eindruck von Schattenrelief zu versehen. Dieses Relief wurde mit dem Luftpinsel geschummert oder, in Fliegerkarten, durch Photographie eines beleuchteten Modells geschaffen. Um zu solchen Modellen zu kommen, bedient man sich heute des «deutschen Konturpantographen ( Dreidimensional-Pantographen», wie er an anderer Stelle genannt wird). Er schneidet mittels einer, in der Höhe veränderlich einzustellenden Schneidnadel aus einem Block von Plastikfolien jeweils in Folienstärke die Konturen aus, so daj am Ende ein Stufenrelief gewonnen wird. Das Verfahren übertrifft an Genauigkeit ein bisher geübtes, bei dem durch elektrisch betätigte Hämmer eine Aluminiumplatte Höhenpunkt für Höhenpunkt vorgetrieben wurde. Ein besonderer «Schattenprojektor», der nach dem telezentrischen bezw. paralleloptischen Prin. zip arbeitet, beleuchtet das Modell, sodaß die Nachteile der bisher üblichen (zentralprojektiven) Beleuchtung mit ihren Verzerrungen des Reliefbildes ausgeschaltet werden. Auch Kopien von solchen Modellen lassen sich in kürzester Zeit herstellen. Dazu wird vorher die mehrfarbige Karte auf eine flache Folie gedruckt (auch dieses Verfahren gehört zu den Neuerungen), diese dann auf das Modell gelegt, erhitzt, worauf sich die Folie dem Modell in allen Feinheiten anlegt. Luftbildes können ebenfalls in dieser Art plastisch umgeformt werden.

Im Druck ist man nicht bei der einen Grauplatte des Reliefs stehen geblieben, sondern hat sich die Vorzüge der «Schweizer Reliefmanier》zunutze gemacht und verwendet neben zwei abgestimmten Grauplatten drei weitere Platten für das Grün der Talstufen und für zwei Brauntöne des höheren Geländes. Auch die Radarnaviga- 
tion fordert eine genauere Darstellung der Bodenformen, wenigstens in Küstennähe. Dies geschieht durch Abschattierungen der Höhenschichten in Grau. Ein weiterer Eindruck bringt, wovon schon die Rede war, die Linien gleicher Differenzen, für Loran Hyperbelscharen, für Shoran konzentrische Kreise. Sie werden mit fluoreszierenden Farben den gewöhnlichen Seekarten aufgedruckt und sind daher nur bei «schwarzem» (ultraviolettem Licht) sichtbar.

In der Photographie hat sich neben Glas und Fiberglas neuerdings auch Sicherheitsglas (《Herculite») eingeführt, das praktisch unzerbrechlich ist. Auf diesem verschiedenen Material ist auch die Glasgravur in mehreren Formen erprobt worden, wobei das Negativ mit einer Licht unwirksam machenden rötlichen bezw. gelben Kollodiumschicht überzogen wird, die für den Graveur das Negativbild schwach durchscheinen läßt. Die einzelnen Farbplatten können auf solche Weise graviert werden und maßhaltig ohne Zwischenschaltung der Kamera direkt zur Kopie auf die Druckplatte verwendet werden. Sogar Druckplatten werden heute mit einer Kolloidschicht überzogen, Polyvinylalkohol mit Bariumsulphat imprägniert. Der Vorteil besteht darin, daß sich auf dieser Schicht mühelos mit Spezialtuschen Zeichnung aufbringen läßt, die direkt zum Druck verwendet werden kann. In Verbindung mit der photolithographischen Übertragung bedeutet das eine wesentliche Erleichterung der Korrekturarbeit. Erwähnt sei noch, da $\beta$ in den Staaten jetzt auch vorbelichtete Offsetplatten im Handel vorrätig gehalten werden.

Auf dem engeren photographischen Sektor ist die Verwendung von Printon-Film von weitreichender Wirkung geworden. Er gestattet die photographische Wiedergabe mehrfarbiger Karten in Direktkopie. Mittels eines anderen, des « Her-Sol»Verfahrens, sind sogar gedruckt vorliegende mehrfarbige Karten ohne Zeichenarbeit, abgesehen von kleineren Retuschen, auf photographischem Wege durch Prismen und Vorschaltung besonderer Filter, in ihre Farben zerlegt, auf kopierfähige Platten zu bringen, soda $\beta$ nach Kopie davon ganze Auflagen gedruckt werden können.

Der Überblick läßt erkennen, vor welchen neuen Möglichkeiten die Kartographie heute steht. Der Weg, der vor 150 Jahren mit Senefelders Erfindung der Lithographie angebahnt wurde und die ausschließliche Herrschaft der Kupferstichkarte durch neue Kartenbilder ablöste, ist noch nicht bis zum Ende beschritten. Wir befinden uns, auch was die inhaltliche Gestaltung der Karte betrifft, wieder im Stadium des Experiments, um die neu eröffneten Möglichkeiten voll auszuwerten. Grund genug deshalb, die weitere Entwicklung mit größtem Interesse zu verfolgen.

Es mu $\beta$ noch angeführt werden, da $\beta$ der vorliegenden Schrift eine ausführliche Bibliographie über alle einsch!ägigen Neuerscheinungen angefügt worden ist. Sie wird damit zu einem nach jeder Richtung hin ergiebigen Nachschlagewerke, unentbehrlich für jeden, der an der Karte schafft oder auch nur sich ihrer vielfältigen Anwendungsmöglichkeiten bedient.

\section{C.ARTOGRAPHIE EN AMÉRIQUE}

Ce premier rapport annuel (1951) de la * Commission on Cartography ", institution fondée en 1941 au Département des Affaires Sociales des Nations Unies, contient les rapports du Brésil, de Canada et des Etats-Unis sur la situation et le progrès de la cartographie officielle. On y trouve encore des "Notices et recherches techniques" sur quelques problèmes actuel de la cartographie moderne, en outre une introduction aux méthodes Radar, qui aujourd'hui sont d'importance non seulement pour la navigation, mais aussi pour les levés topographiques. Appareils et instruments nouveaux de l'aérophotogrammétrie montrent de larges possibilités, l'introduction des feuilles plastiques a conduit à des changements révolutionnaires du dessin et du tirage des cartes. Le rapport s'achève par une bibliographie très informée des nouveautés cartographiques.

\section{LA CARTOGRAFIA IN AMERICA}

Il primo rapporto annuale (1951) nella „Commission on Cartography “, istituzione fondata nel 1941 dal Dipartimento degli aftari sociali delle Nazioni Unite, contiene il rapporto del Brasile, del Canadà e degli Stati Uniti sulla situazione e sul progresso della cartografia ufficiale. Oltre alle notizie 
e ricerche tecniche sui problemi attuali della cartografia moderna, c'è una introduzione interes. sante sui singoli metodi Radar, che oggi non hanno solo una importanza per la navigazione, ma progressivamente anche per $\mathrm{i}$ rilievi topografici. Nuovi istrumenti aerofotogrammetrici mostrano grandi possibilità di sviluppo, l'introduzione dei fogli plastici ha portato un capovolgimento nel disegno e nella stampa e lasciano intravedere altre novità. Una dettagliata bibliografia delle novità cartografiche chiude il rapporto.

\section{DAS NABELORNAMENT DER „AHNENFIGUREN“ AUF DEN ZEREMONIALSTÜHLEN VOM SEPIK, NEUGUINEA \\ Alfred SteinmanN}

Im Rahmen der Beschreibung eines im Bernischen Historischen Museum befindlichen Zeremonialstuhles vom Sepikgebiet (Abb.I) iveist E. RoHRER ${ }^{1}$ auf die eigenartige, an der Nabelgegend der Hauptfigur vorhandene Verzierung hin. Sie wird als ein „sternförmig eingeritztes Muster.... das wohl ein Narbenornament darstellt, aber gerade am lebenden Menschen nicht häufig zu finden ist, “ beschrieben, welches auch auf anderen Gegenständen vom Sepik wie z. B. als Nabelverzierung menschlicher Figuren von zwei Aufhängehaken sowie auf der Seitenfläche eines Schwirrholzes anzutreffen sei. Ein ähnliches, ebenfalls als Nabelornament menschlicher Figuren auf Kultstühlen von Neuguinea auftretendes, wenn auch stark vereinfachtes Muster ist von J, SöDERSTRÖ ${ }^{2}{ }^{2}$ erwähnt worden. Er bringt es mit dem in Indonesien und Ostasien verbreiteten Rosettenmuster in Beziehung.

Bei Betrachtung der von ROHRER abgebildeten Zeichnungen (Abb. 1, a-d) fallen zwei voneinander etwas abweichende Ornamentkombinationen auf. Bei Abb. $1 \mathrm{a}$ und $\mathrm{b}$ besteht das Ornament aus einem kreuzförmig um eine zentrale, kreisrunde Scheibe angeordneten Gebilde, dessen vier Strahlen, Auswüchse oder "Arme" je in einem "W "-förmigen Zeichen endigen. Von diesen weichen die Ornamente auf $\mathrm{Abb} .1 \mathrm{c}$ und $\mathrm{d}$ etwas ab, Sie lassen trotzdem ihre Verwandtschaft mit den zwei vorhergehenden erkennen. Bei ihnen erscheint das kreuzförmige Gebilde in etwas abgewandelter und vereinfachter Form als Raute, an deren spitzen Enden das „W"-förmige Zeichen mehrmals hintereinander wiederholt worden ist, während die mittlere Scheibe fehlt.

Wir glauben dieses Nabelornament nicht vom Rosettenmotiv ableiten zu dürfen, sondern im Gegenteil nachweisen zu können, daß dessen Herkunft anderswo gesucht werden muß. Bei den «W》förmigen Zeichen handelt es sich nämlich, wie $C$. SCHUSTER ${ }^{3}$ seinerzeit nachweisen konnte, um die auf dem asiatischen Festland und im westlichen Pazifikgebiet weitverbreitete, wenn auch stark stilisierte Wiedergabe eines in der Regel als Fregattvogel bezeichneten, im Fluge dargestellten Vogels, die in den verschiedensten Varianten vornehmlich als Tatauierungszeichen in Erscheinung tritt. Besonders charakteristisch ist dabei eine gewisse Art der Darstellung, bei welcher vier « $W$ » förmige oder auch manchmal umgekehrt 《W 》förmige, stilisierte Vögel darstellende Zeichen, kreuzweise an den Spitzen oder Ecken eines rautenförmigen Gebildes auftreten ( $A b b .1 \mathrm{c}$ und d, Abb. $6 \mathrm{a}, \mathrm{b}, \mathrm{c}$ ). In ihrer kompliziertern Form mit Kreuzmuster und zentraler Scheibe (Abb. 1 a und b) zeigt diese Ornamentkombination eine bemerkenswerte Ähnlichkeit mit Ziermustern auf jenen unter dem Namen «Kapkap 》 bekannten melanesischen Schmuckplatten, die aus einer kreisrunden, gewöhnlich aus der Schale der Tridacnamuschel (Tridacna gigas) geschliffenen weißen Kalkscheibe bestehen, auf der eine verschiedenartige, aus hell- bis dunkelbraunem Schildpatt «à jour 》 ausgeschnittene Zierauflage befestigt ist (Abb. 2). Diese Ähnlichkeit tritt beim Vergleich der hier abgebildeten Kapkapscheibe von den zum Bismarck-Archipel gehörenden Admiralitätsinseln mit dem Nabelornament von Abb. 1 b (Sepik, Neuguinea) besonders deutlich zutage;

1 E. Rohrer: Ein Zeremonialstuhl von Sepik (Neuguinea). (Bull. d. Schweiz. Ges.. f. Anthropologie und Ethnologie, 28. Jahrg. 1951/52, Bern 1952, Seite 39-48).

2 J. Söderström: Die Figurstühle vom Sepikfluß auf Neuguinea. (Statens Etnografiska Museum, Smärre meddelanden Nr. 18, Stockholm 1941, S. 5-44, Abb. 8 a und 8b), jener G. HöltKER: Der Donnerkeilglaube vom steinzeitlichen Neuguinea aus gesehen. (Acta Tropica, I, 1. 1944, Abb. 10, S. 47).

${ }^{8}$ C. SChUster: On bird-designs in the Western Pacific: Indonesia-Melanesia-Polynesia. (Cultureel Indië, I. 1939, Leiden, S. 232-235). 